\title{
Response dynamics in prospective memory
}

\author{
Drew H. Abney • Dawn M. McBride • Angela M. Conte • \\ David W. Vinson
}

Published online: 19 November 2014

(C) Psychonomic Society, Inc. 2014

\begin{abstract}
Prospective memory (PM) is the ability to remember to execute a delayed behavior. Most theoretical and empirical work on PM has focused on the attentional resources that might facilitate successfully executing a delayed behavior. In the present study, we enhance the current understanding of attention allocation and also introduce novel evidence for the dynamics of PM retrieval. We recorded mouse-tracking trajectories during a prospective memory task to examine the continuous nature of attentional processes that support PM cue retrieval. We found that the velocity profiles of response trajectories differed as a function of PM cue focality while controlling for the canonical measure of response time, supporting the notions that monitoring is evident in the continuous nature of response trajectories and that such trajectories are sensitive to cue focality. Conditional velocity profiles of ongoing task trials indicated that monitoring occurred when the processing of PM cues differed from ongoing task instructions (Nonfocal PM condition): responses were made later in the profile, suggestive of a more controlled retrieval process. Analysis of PM cue retrieval profiles indicated correctly retrieved Focal PM cues were qualitatively and quantitatively different from all other PM cue retrieval trials. This provides evidence that retrieval dynamics of a delayed behavior differ as a function of cue focality and suggests that controlled processing may contribute to spontaneous retrieval of a PM task.
\end{abstract}

D. H. Abney $(\bowtie) \cdot$ D. W. Vinson

Cognitive and Information Sciences, University of California,

Merced, CA, USA

e-mail: drewabney@gmail.com

D. M. McBride $\cdot$ A. M. Conte

Department of Psychology, Illinois State University, Normal, IL, USA
Keywords Prospective memory $\cdot$ Mouse-tracking $\cdot$ Temporal dynamics

\section{Introduction}

Prospective memory (PM) is the ability to remember to execute delayed intentions. Examples of PM include remembering to pay a bill before the due date and remembering to take medication at a certain time in the day. One debated question is the amount of attention required for successful PM. Previous work suggests that successful PM retrieval is supported by processes such as attentional monitoring (Smith, 2003), cue-driven spontaneous retrieval (Einstein \& McDaniel, 2005), or a dynamic combination of both processes (Scullin, McDaniel, \& Shelton, 2013). In the present study, we introduce a new method for examining the various processes potentially facilitating PM performance.

In the more commonly used laboratory PM paradigm, participants complete an ongoing task in the first block of a two-block experiment. After completing the first block, but before the second block, participants either continue with the ongoing task alone or continue with the ongoing task with the additional instruction that if some specified event occurs (i.e., PM cue), they are to make a particular response. This affords examination of the amount of attention required for different PM task conditions. Typically, this inquiry is informed by identifying average response time (RT) differences between block 1 and block 2 for the different PM task conditions. If RT is higher in block 2 for conditions where a PM task was provided, then PM researchers infer that additional attentional costs occurred beyond those required for the ongoing task (Smith, 2003).

Two different processes that might support PM retrieval are attentional monitoring and spontaneous retrieval. Attentional monitoring (henceforth, monitoring) during a PM task refers 
to an effortful maintenance of the PM intention to attend to cues in the environment that indicate when the PM response should be made (Smith, 2003). Monitoring is typically evidenced by longer RTs and/or lower accuracy for ongoing task performance when a PM task is activated. The preparatory attentional and memory (PAM) processes view of PM (Smith, 2003) proposes that PM performance cannot rely solely on automatic processing, and, thus, attentional processes are required for successful PM performance. Therefore, according to the PAM view, longer RTs should accompany successful $\mathrm{PM}$ retrieval.

Spontaneous retrieval is a process that supports the retrieval of a delayed behavior without requiring additional attentional resources (McDaniel, \& Einstein, 2007). The Multiprocess (MP) view (McDaniel \& Einstein, 2000) proposes that the utilization of processes such as monitoring and spontaneous retrieval, are influenced by context - properties of the ongoing task and the PM tasks - namely, cue focality (Einstein \& McDaniel, 2005).

The current study focused primarily on cue focality. In general, a PM task can be either focal or nonfocal. A focal PM task encompasses ongoing and PM tasks that share processing features. For example, if the ongoing task is a lexical decision task ("is this stimulus a WORD/NONWORD?"), the PM task might be to search for specific words. A nonfocal PM task encompasses ongoing and PM tasks that reflect different types of processing, for example, searching for words with a particular number of syllables within an ongoing lexical decision task. The PAM view predicts attentional monitoring in both focal and nonfocal PM tasks (Smith, 2003). The MP view predicts monitoring during nonfocal PM tasks, and other types of processing such as spontaneous retrieval, during focal PM tasks. Although much work has advanced our current understanding of the contextual factors and processes that are associated with PM, there is still debate regarding when processes such as monitoring and spontaneous retrieval are utilized (Einstein \& McDaniel, 2010; Smith, 2010, also see Scullin et al., 2013).

\section{Current study and hypotheses}

The current study addressed two related goals. First, we addressed the current debate regarding the utilization of attentional processing in PM tasks by introducing a method new to the PM literature: the analysis of response dynamics for computer mouse-tracking. Mouse-tracking techniques provide a novel way to investigate response dynamics in forced choice decision-making tasks (Freeman, Dale, \& Farmer, 2011) such as lexical decision. Dynamic information about the decisionmaking process, such as mouse position, velocity, and acceleration, can be captured using two-dimensional (2D) computer mouse movements (Freeman \& Ambady, 2010).
Our analysis focused primarily on the velocity profiles of mouse trajectories toward response choices. Velocity measurements show the rate of change in the position of mouse movements throughout the response trajectory. These are simple continuous measures that map onto the raw movements of mouse positions. Evidence of monitoring in all PM tasks would support the PAM view. In the response dynamics, this would correspond to different velocity profiles for PM conditions relative to a control condition where no PM task was given. Specifically, observations of peak velocity later in the response trajectory for all PM conditions, relative to a control condition, would be indicative of more controlled processes, e.g., monitoring. The MP view, however, suggests that cue focality influences the use of attentional processes. Thus, observations of different response dynamics as a function of cue focality are predicted by the MP view. Specifically, we would expect peak velocities later in response trajectories for the nonfocal PM condition and no differences between focal PM and control conditions. Observations of spontaneous retrieval would provide additional support for the MP view. Spontaneous retrieval and PM cue retrieval were addressed by the second goal of this study.

The second goal of the current study was the investigation of the retrieval component of PM. With our design, we were able to examine the response dynamics of the noticing or retrieval of the PM cue in addition to the typical question of PM cue response performance. In other words, our second goal examined the question: What are the response dynamics when indicating that a PM cue (e.g., HORSE or TIGER) is a WORD and do these dynamics differ as a function of the subsequent PM cue response performance (Hit vs. Miss) or cue focality (Focal vs. Nonfocal)?

In laboratory-based PM studies, participants are instructed to respond to a PM cue by performing a specific action (e.g., press the "/" key). Although some previous work examined RT differences in the responses to PM cues (Brewer, Knight, Marsh, \& Unsworth, 2010; Marsh, Hicks, \& Watson, 2002), this component of the PM task has received little attention in the literature. In Marsh et al.'s proposal of a microstructure of prospective memory, they observed that successfully noticed PM cues were processed slower relative to control words. Brewer et al. (2010) observed that low-working-memory participants correctly responded slower to the first nonfocal cue relative to high-working-memory participants. They termed this slower responding cue interference. These results point to cue interference when the PM intention is activated; however, interference has also been observed during the suspension of the PM intention and is suggested to correspond to intention interference (Scullin, Einstein, \& McDaniel, 2009).

Considering previous research on the noticing of PM cues, we expected to observe longer RTs for correctly retrieved PM targets $\left(\mathrm{PM}_{\mathrm{HIT}}\right)$ relative to $\mathrm{PM}$ targets that went unnoticed ( $\left.\mathrm{PM}_{\mathrm{MISS}}\right)$, independent of cue focality. Consistent with the 
predicted RT results, we also expected to observe response dynamics for $\mathrm{PM}_{\mathrm{HIT}}$ trials that were qualitatively and quantitatively different to $\mathrm{PM}_{\mathrm{MISS}}$ trials and consistent with a view that processing is undergoing cue interference. Specifically we expected to observe peak velocities later in the trajectory or narrower peaked velocity profiles for $\mathrm{PM}_{\mathrm{HIT}}$ trials relative to $\mathrm{PM}_{\mathrm{MISS}}$ trials. A narrow-peaked velocity profile suggests the absence of a distinct response; a defined peak somewhere in the central area of a profile typically indicates the latter. Although there are some similarities between the evidence for cue interference and monitoring, the difference between them is in the conceptual and analytic treatment of ongoing task trials versus PM cue trials.

Hypotheses regarding the response dynamics for PM cue trials as a function of focality condition are less clear. One possibility is that we might observe differences as a function of cue focality such that more cue interference occurs for Focal $_{\text {HIT }}$ trials (i.e., focal cue trials where a correct PM response is made after the trial) relative to Nonfocal $_{\text {HIT }}$ trials (i.e., nonfocal cue trials where a correct PM response is made after the trial). This result could be due to the spontaneous retrieval process that we expect for focal PM cues. As described by McDaniel, Guynn, Einstein, and Breneiser (2004), spontaneous retrieval can occur through a cue-focused discrepancy attribution process. In other words, the PM cue can be noticed based on the discrepancy of its significance compared with other ongoing task stimuli. Then an attribution process allows for retrieval of the PM task intention requiring the diverting of attention from the ongoing task and resulting in a slower response dynamic for Focal ${ }_{\text {HIT }}$ trials than Nonfocal $_{\text {HIT }}$ trials (where monitoring processes are more likely to support successful PM task retrieval). ${ }^{1}$ However, this hypothesis is speculative, given the novel procedure employed in the current study.

\section{Method}

Participants and design

Seventy-three undergraduates at the University of California, Merced participated and received course credit. All of the participants were fluent English speakers. Participants who either failed to recall PM cues at the end of the experiment or did not follow task instructions indicated by, at the end of the experiment, failing to remember the instruction to press " $\mathrm{z}$ " when presented with a PM cue $(n=10)$ or where a computer/ mouse error occurred $(n=1)$, were excluded from analyses. Remaining participants were randomly assigned to one of three conditions: Control ( $n=24)$, Focal $(n=17)$, Nonfocal $(n=21)$.

\footnotetext{
${ }^{1}$ We thank Michael Scullin for this suggestion.
}

\section{Materials}

Stimulus presentation procedures were controlled by MouseTracker (Freeman \& Ambady, 2010). Stimuli consisted of uppercase black letter strings in 40-point Arial font that appeared in the center of a white screen. We chose 56 words from the Kučera and Francis (1967) normative compendium and 60 nonwords from the ARC Nonword Database (Rastle, Harrington, \& Coltheart, 2002). All words and nonwords were matched for frequency and letter range (four to six). PM cue stimulus properties (i.e., animals) were not used in the stimulus set. The PM cues were two animal words, "HORSE" and "TIGER."

\section{Procedure}

Participants received instructions for a lexical decision task: decide if a string of letters was a word or not. Each trial began with a "START" button at the bottom-center (width $=.2$, height $=.1, \mathrm{x}=0, \mathrm{y}=.1)^{2}$ of the screen and the two decision buttons (width $=.3$, height $=.2)$ at the top left $(\mathrm{x}=-1, \mathrm{y}=1.5)$ and top right $(\mathrm{x}=.7, \mathrm{y}=1.5)$ corners of the screen. To begin the trial, participants were instructed to click the "START" button, which provided a consistent set point for cursor position. After clicking the "START" button, the trial screen was immediately presented with the stimulus in the center of the screen. Once the trial screen was presented, participants were instructed to move their mouse and click on either the "WORD" or "NONWORD" decision button. Response mapping of the decision buttons was counterbalanced across participants. After the subject's response, a text response window appeared and participants were instructed to "Press Enter to Proceed to Next Trial", to continue to the next trial. See Fig. 1 for task set-up.

Participants completed four practice trials before beginning the baseline block of trials. All participants received the same stimuli in the baseline block in the same random order. The baseline block consisted of 30 words and 30 nonwords. After completing the baseline block, different PM block instructions were given to participants depending on condition. Participants in the focal condition were instructed to continue with the lexical decision task. In addition, if the words "HORSE" or "TIGER" (Focal PM cues) appeared, they were to enter " $\mathrm{z}$ " into the text response window (PM task response) after completing the trial. Participants in the nonfocal condition were instructed to continue with the lexical decision task. In addition, if an animal word (Nonfocal PM cues) appeared, they were to enter " $\mathrm{z}$ " into the text response window after completing the trial. The same two PM cues were used for the focal and nonfocal conditions and each was presented twice in

\footnotetext{
${ }^{2}$ All button properties and coordinates are in MouseTracker coordinate space dimensions (see Freeman \& Ambady, 2010).
} 


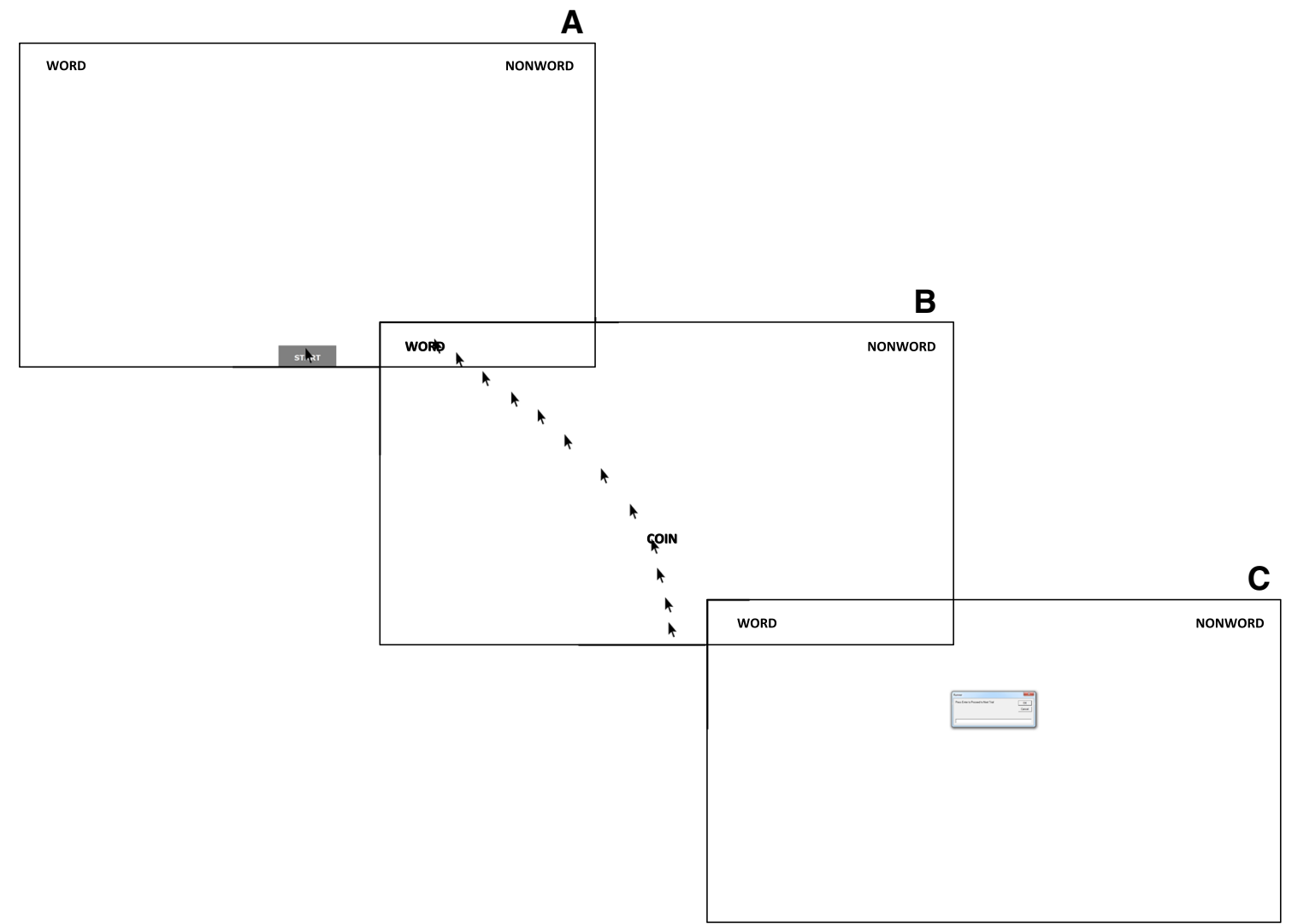

Fig. 1 Screen set-up of task. (A) Trial begins when a participant presses the START button. (B) Participants move their mouse cursor to either the WORD or NONWORD button. (C) After response selection, a text response window appears (also occurred during practice trials) and

a fixed order (trials 31, 38, 47, 57). Participants in the control condition were instructed to continue with the lexical decision task. At this time, participants were allowed to ask the experimenter any questions about the new task instructions.

After being presented with the PM block instructions, all participants completed a short distracter task for 3 min. After completion of the distracter task, but before proceeding with the PM block, participants were reminded of the lexical decision task instructions but not of the PM task instructions. PM block stimuli were taken from the same databases as in the baseline block ( 26 words and 30 nonwords). All other stimulus presentations and response procedures were identical to those in the baseline block. Upon completion of the PM block, participants were asked to recall task instructions.

\section{Results}

For all analyses, alpha was set to .05 and multiple comparisons were subject to Bonferroni corrections. Response dynamics were recorded and mouse-tracking measures participants are instructed to "Press Enter to Proceed to Next Trial." Prospective memory (PM) retrieval can occur during this component of the trial

were computed in the MouseTracker software. Trials were discarded from analysis $(7.5 \%)$ if the response was not made within 3,000 ms (RT) or if initial movements (initiation time; IT) began more than $400 \mathrm{~ms}$ after stimulus onset (c.f. Papesh \& Goldinger, 2012). All analyses were performed only on correct responses to the "WORD" decision button, i.e., no analyses were conducted on responses to the "NONWORD" decision button nor on PM cue trials unless otherwise specified (see Analysis of PM cue retrieval sub-section).

\section{PM cue response performance}

PM cue detection was defined as the proportion of the four cues with accurate responses. PM cue responses were considered correct if participants entered " $z$ " into the text response box after responding to the trial. A one-way ANOVA was performed on PM accuracy data with PM condition (Focal vs. Nonfocal) as a fixed factor and showed no reliable difference between Focal $(M=.71, S E=.08)$ and Nonfocal $(M=.64, S E$ $=.09) \mathrm{PM}$ cue response performance, $F(1,39)=.245, p=$ $.623, \eta_{\mathrm{p}}^{2}=.007$. 
Ongoing task accuracy

Hereafter, unless otherwise noted, all hypothesis testing included an ANCOVA on PM block measures with Baseline block measures as covariates and PM condition (Control, Focal, and Nonfocal) as the fixed factor. Mean accuracy proportions on the ongoing task for baseline and PM blocks are presented in Table 1. There were no reliable differences across conditions for ongoing task accuracy, $F(1,58)=.245, p$ $=.58, \eta_{\mathrm{p}}^{2}=.020$.

Ongoing task response times and initiation times (ITs)

Mean ongoing task RTs for baseline and PM blocks are presented in Table 1. For this task, RT corresponds to the duration between when the stimulus was presented and when a mouse click was made on the response button. A significant effect of PM condition, $\left(F[2,58]=6.07, p=.004, \eta_{\mathrm{p}}{ }^{2}=.173\right)$, indicated that mean RTs for the Control condition were faster than RTs for the Nonfocal condition, $p=.003$. No other differences were reliable, $p \mathrm{~s} \geq .11$.

ITs were computed using the MouseTracker analyzer. IT corresponds to the duration between when the stimulus was presented and when the first mouse movement was executed. Mean ITs did not significantly differ across PM Conditions, $F(2,58)=1.28, p=.286, \eta_{\mathrm{p}}{ }^{2}=.042$.

\section{Response dynamics of ongoing task trials}

Velocity profiles were computed to examine the attentional mechanisms employed for successful PM retrieval in the different PM tasks over the course of the entire response trajectory. We generated 20 normalized time bins of the average velocity (VEL) for the Euclidean distance (xy-axes) toward the "WORD" decision button for each correct trial. Therefore, we were able to detect changes in velocity at different stages of a trial in normalized space for each condition. Euclidean distance velocity profiles corresponded to the temporal derivative of $\mathrm{x}$ - and $\mathrm{y}$-axis positions toward the "WORD" decision button. A relative increase or decrease in velocity toward a decision button can be informative about where in the movement trajectory for different PM conditions a change in speed occurs for the response. VEL was computed for each participant separately for the baseline and PM blocks. We also computed the maximum velocity $\left(\mathrm{MAX}_{\mathrm{VEL}}\right)$ for each trial and in which bin $\mathrm{MAX}$ VEL occurred $\left(\mathrm{MAX}_{\mathrm{VELBIN}}\right)$.

We conducted two different tests: (1) an ANCOVA on VEL for the PM block for each time bin with PM task condition as the fixed factor and baseline block average VEL as the covariate to determine conditional effects of PM task, and (2) ANCOVAs on $\mathrm{MAX}_{\mathrm{VEL}}$ and MAX $\mathrm{VELBIN}_{\mathrm{V}}$ for the PM block, with PM task condition as the fixed factor and Baseline block $\mathrm{MAX}_{\mathrm{VEL}} / \mathrm{MAX}_{\mathrm{VELBIN}}$ and PM block RT as the covariates to determine if response dynamics can predict PM condition, controlling for the canonical measure of RT.

Across-condition covariate models of average velocity (VEL) Figure 2 displays the covariate models of aggregated velocity profiles in the PM block for each condition. A significant effect of the fixed factor was observed for time bins 8-9 and 13-17. Post-hoc analyses suggested two distinct trajectory phases during responses across PM conditions: increasing VEL peaking at time bin 9 (phase one: bins 8-9) followed by a decrease in VEL (phase two: bins 13-17). See Table 2 for multiple comparisons of all significant ANCOVA fixed effects. During phase one, the Focal condition had a higher average VEL than both the Nonfocal and Control conditions. During phase two, the Nonfocal condition had a higher VEL than both the Focal and Control conditions. ${ }^{3}$

Across-condition covariate models of $M A X_{V E L}$ and $M A X_{V E L B I N}$ Mean $\mathrm{MAX}_{\mathrm{VEL}}$ and MAX $\mathrm{VELBIN}$ for baseline and PM blocks are presented in Table 1. Mean MAX ${ }_{\mathrm{VEL}}$ did not differ significantly across PM Conditions, $p s \geq .97$. For $\mathrm{MAX}_{\mathrm{VELBIN}}$, a main effect of PM Condition, $F(2,57)=$ $3.48, p=.037, \eta_{\mathrm{p}}{ }^{2}=.109$, and pairwise comparisons, indicated that, controlling for RT, MAX $\mathrm{VEL}_{\mathrm{VE}}$ occurred later in the normalized trajectory space for the Nonfocal PM Condition relative to the Focal PM Condition, $p=.033$. No other effects were significant, $p s \geq .334$.

\section{Analysis of PM cue retrieval}

To assess as many PM cue retrieval trials as possible, there was no filtering of trial by RT and IT as was done for the ongoing task trials. Mean RT and IT were computed for each trial separately for the four PM cue retrieval trial types: Focal $_{\mathrm{HIT}}$, Focal ${ }_{\mathrm{MISS}}$, Nonfocal $\mathrm{HIT}_{\mathrm{HI}}$, and Nonfocal ${ }_{\text {MISS. "Hit" corresponded to a successful }}$ PM cue retrieval trial and a "Miss" corresponded to an unsuccessful PM cue retrieval trial. Due to variable performance for each participant, all PM cue retrieval measures were computed for each trial, which is

\footnotetext{
${ }^{3}$ Dale, Kehoe, and Spivey (2007) simulated random trajectories for 101 time bins and provided a criterion of eight and six consecutive significantly different $t$ tests for an alpha criterion of .01 and .05 , respectively. Therefore, considering our 20 time-bin mouse trajectories, we set a criterion of 1.58 and 1.18 consecutive significantly different $t$ tests for an alpha criterion of .01 and .05 , respectively. For the sake of simplicity and to be conservative, we considered statistical significance across two consecutive bins as our criterion for a reliable difference across conditional velocity profiles.
} 
Table 1 Ongoing task accuracy, ongoing task response time (RT) (in ms), ongoing task initiation time (IT) (in ms), maximum velocity $\left(\mathrm{MAX}_{\mathrm{VEL}}\right)$, in which bin $\mathrm{MAX}_{\mathrm{VEL}}$ occurred $\left(\mathrm{MAX}_{\mathrm{VELBIN}}\right)$, and standard error (in parentheses) by prospective memory (PM) task condition, Block, and PM cue retrieval trial type

\begin{tabular}{|c|c|c|c|c|c|}
\hline & Ongoing Task Accuracy & Mean RT & Mean IT & $\mathrm{MAX}_{\mathrm{VEL}}$ & MAX $_{\text {VELBIN }}$ \\
\hline \multicolumn{6}{|l|}{ Control } \\
\hline Baseline & $.98(.005)$ & $1605(46)$ & 192(13) & $.057(.003)$ & $10.7(.22)$ \\
\hline PM & $.99(.003)$ & $1458(56)$ & $185(13)$ & $.058(.003)$ & $10.5(.31)$ \\
\hline \multicolumn{6}{|l|}{ Focal } \\
\hline Baseline & $.98(.007)$ & $1563(54)$ & $221(15)$ & $.058(.003)$ & $10.5(.26)$ \\
\hline PM & $.99(.004)$ & $1490(66)$ & 194(16) & $.060(.004)$ & $9.7(.37)$ \\
\hline \multicolumn{6}{|l|}{ Nonfocal } \\
\hline Baseline & $.97(.006)$ & 1616(49) & $209(14)$ & $.054(.003)$ & $10.9(.24)$ \\
\hline PM & $.99(.003)$ & $1676(60)$ & $215(14)$ & $.056(.003)$ & $11.3(.33)$ \\
\hline \multicolumn{6}{|l|}{ PM cue retrieval } \\
\hline Focal $_{\text {HIT }}$ & - & $3028(339)$ & $186(43)$ & $.134(.007)$ & $10.0(.55)$ \\
\hline Focal $_{\text {MISS }}$ & - & $2280(526)$ & $328(68)$ & $.120(.011)$ & $8.2(.86)$ \\
\hline Nonfocal $_{\text {HIT }}$ & - & 1976(320) & $341(41)$ & $.107(.007)$ & $10.5(.52)$ \\
\hline Nonfocal $_{\text {MISS }}$ & - & $2301(437)$ & $308(56)$ & $.139(.009)$ & $9.6(.71)$ \\
\hline
\end{tabular}

different from the by-participant level measures computed for the ongoing task analyses reported above. Mean RTs and ITs for PM cue retrieval trial types are presented in Table 1. Separate 2 (PM condition: Focal vs. Nonfocal) $\times 2$ (Success: Hit/Miss) ANOVAs with RT and IT as dependent measures suggested no reliable differences, $p \mathrm{~s}>.101$.

Response dynamics of PM cue retrieval Mean MAX $\mathrm{VEL}_{\mathrm{V}}$ and $M_{\text {AELBIN }}$ were computed for each trial and separately for the four PM cue retrieval trial types. A 2 (PM condition: Focal vs. Nonfocal) $\times 2$ (Success: Hit/Miss) ANOVA with MAX ${ }_{V E L}$ as the dependent measure showed a significant interaction, $F(1,147)=6.73, p=.01$, and pairwise comparisons suggested that $\mathrm{MAX}_{\mathrm{VEL}}$ for Nonfocal $_{\mathrm{HIT}}(M=.11, S E=.007)$ was smaller than Focal HIT $(M=.13, S E=.007)$ and Nonfocal $_{\text {MISS }}(M=.14, S E=.009), p s<.01$. No other effects were significant, $p \mathrm{~s}>$.165. A 2 (PM condition: Focal vs. Nonfocal) $\times 2$ (Success: Hit/Miss) ANOVA with MAX $_{\text {VELBIN }}$ as the dependent measure showed a main effect of Success, $F(1,147)=5.28, p=.04$, suggesting that $\mathrm{PM}_{\mathrm{HIT}}$ $(M=10.29, S E=.38)$ reached $\mathrm{MAX}_{\mathrm{VEL}}$ later in the velocity profile relative to $\mathrm{PM}_{\mathrm{MISS}}(M=8.89, S E=.56)$. All other effects were not significant, $p \mathrm{~s}>.289$.

Velocity profiles of PM retrieval cue trial type were computed to determine if the dynamics of PM cue retrieval differed as a function of PM cue focality (Focal/Nonfocal) and success (Hit/Miss). VEL was

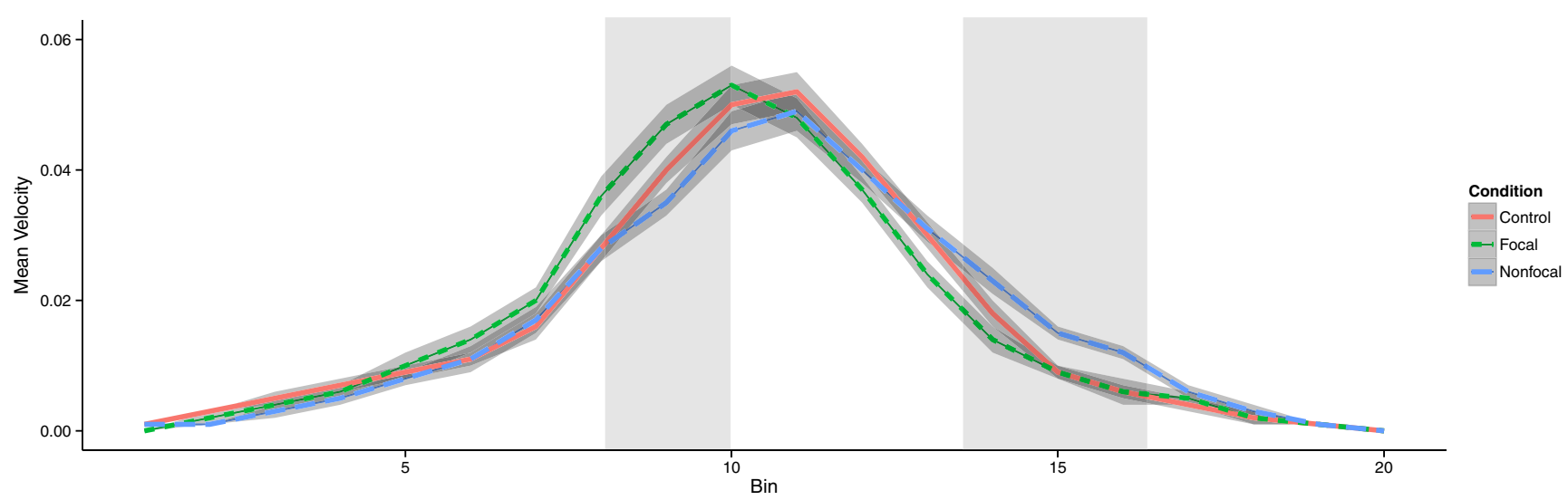

Fig. 2 Block 2 covariate model for all three conditions. Width of lines indicates $( \pm)$ standard error. Vertical bars indicate significant fixed effects $(\geq 2$ bins as per criterion) 
Table 2 Multiple comparisons of covariate model for bins with statistically significant fixed effects

\begin{tabular}{lllllllllllllll}
\hline & $\operatorname{Bin} 4$ & $\operatorname{Bin} 5$ & $\operatorname{Bin} 6$ & $\operatorname{Bin} 7$ & $\operatorname{Bin} 8$ & $\operatorname{Bin} 9$ & $\operatorname{Bin} 10$ & $\operatorname{Bin} 11$ & $\operatorname{Bin} 12$ & $\operatorname{Bin} 13$ & $\operatorname{Bin} 14$ & $\operatorname{Bin} 15$ & $\operatorname{Bin} 16$ & $\operatorname{Bin} 17$ \\
\hline $\mathrm{C}$ vs. F & $n s$ & $n s$ & $n s$ & $n s$ & $*$ & $\dagger$ & $n s$ & $n s$ & $n s$ & $\dagger$ & $n s$ & $n s$ & $n s$ & $n s$ \\
$\mathrm{C}$ vs. NF & $n s$ & $n s$ & $n s$ & $n s$ & $n s$ & $n s$ & $n s$ & $n s$ & $n s$ & $n s$ & $*$ & $* *$ & $* *$ & $\dagger$ \\
$\mathrm{F}$ vs. NF & $n s$ & $n s$ & $n s$ & $n s$ & $*$ & $* *$ & $n s$ & $n s$ & $n s$ & $*$ & $* *$ & $* *$ & $* *$ & $n s$ \\
\hline
\end{tabular}

Note. $\dagger p \leq .1, * p \leq .05, * * p \leq .01, * * * p \leq .001 . \mathrm{C}=\mathrm{Control}, \mathrm{F}=$ Focal, $\mathrm{NF}=$ Nonfocal, $\mathrm{ns}=$ not significant

computed for each trial separately for the four PM retrieval cue trial types. Figure 3 displays the aggregated velocity profiles for the four PM cue retrieval trial types. Paired-sample t-tests were conducted on each bin for each combination of PM retrieval cue trial type. See Table 3 for all significant effects by bin. Again, we used a conservative criterion of two or more consecutive bins below $p<.05$ to consider differences of velocity profiles as reliably different.
Taken together, the results from these analyses suggested that the Focal ${ }_{\text {HIT }}$ VEL profiles were reliably different from the Focal $_{\text {MISS }}$ and Nonfocal ${ }_{\text {HIT }}$ VEL profiles. Qualitatively, the aggregated VEL profiles for Focal HIT $_{\text {were distinct relative to }}$ all other PM cue retrieval VEL profiles. Furthermore, the other PM cue retrieval VEL profiles were qualitatively similar to the ongoing task VEL profiles with respect to the similar two-phase trajectory of increasing VEL to a peak, and then decreasing VEL.
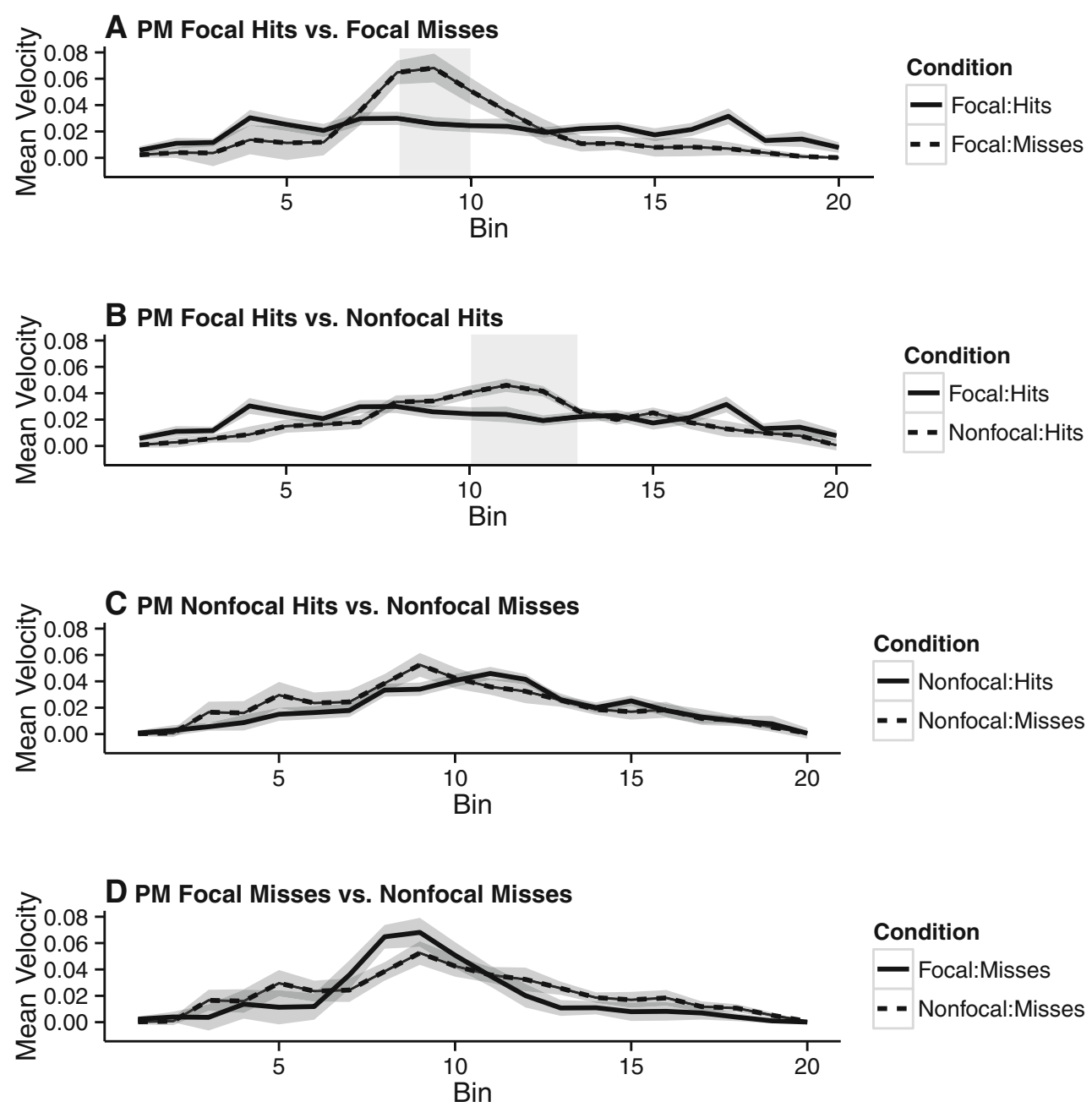

Fig. 3 Velocity profiles of prospective memory (PM) trial type comparisons. (A) PM focal hits versus focal misses. (B) PM focal hits versus nonfocal hits. (C) PM nonfocal hits versus nonfocal misses. (D) PM focal misses versus nonfocal misses. Width of lines indicates $( \pm)$ standard error. Vertical bars indicate significant fixed effects ( $\geq 2$ bins as per criterion) 
Table 3 Effects of paired-sample t-tests of prospective memory (PM) trial type combinations

\begin{tabular}{lllllllllllllll}
\hline & Bin 4 & Bin 5 & Bin 6 & Bin 7 & Bin 8 & Bin 9 & Bin 10 & Bin 11 & Bin 12 & Bin 13 & Bin 14 & Bin 15 & Bin 16 & Bin 17 \\
\hline F:H vs. F:M & $n s$ & $n s$ & $n s$ & $n s$ & $* *$ & $* *$ & $n s$ & $n s$ & $n s$ & $*$ & $\dagger$ & $n s$ & $n s$ & $n s$ \\
F:H vs. NF:H & $* *$ & $n s$ & $n s$ & $\dagger$ & $n s$ & $n s$ & $*$ & $* *$ & $* * *$ & $n s$ & $n s$ & $n s$ & $n s$ & $*$ \\
NF:H vs. NF:M & $n s$ & $n s$ & $n s$ & $n s$ & $n s$ & $*$ & $n s$ & $n s$ & $n s$ & $n s$ & $n s$ & $n s$ & $n s$ & $n s$ \\
F:M vs. NF:M & $n s$ & $n s$ & $n s$ & $n s$ & $\dagger$ & $n s$ & $n s$ & $n s$ & $n s$ & $*$ & $n s$ & $n s$ & $n s$ & $n s$ \\
\hline
\end{tabular}

Note. $\dagger p \leq .1,{ }^{*} p \leq .05,{ }^{*} p \leq .01, * * * p \leq .001 . \mathrm{F}=$ Focal, $\mathrm{NF}=$ Nonfocal, $\mathrm{H}=\mathrm{Hit}, \mathrm{M}=$ Miss, $\mathrm{ns}=$ not significant

\section{Discussion}

The results of the current study provide insight into the current debate regarding attentional processing and cue focality in PM performance (Einstein \& McDaniel, 2010; Smith, 2010). Investigation of the response dynamics provided additional support for the MP view and a unique look into the dynamics of PM cue retrieval.

\section{Attentional processes in PM}

We first address the issue of controlled versus automatic processes in successful PM performance. Although we did not show evidence for PM performance differing as a function of cue focality, ${ }^{4}$ it is possible that high PM performance in the nonfocal condition was facilitated due to utilizing controlled processes, such as monitoring. Observing longer RTs in the nonfocal condition relative to the control condition supported this interpretation.

Further support comes from the results of the response dynamics. Differences in the velocity profiles and MAX $_{\text {VELBIN }}$ suggest that participants in the nonfocal condition increased in VEL later on in the response trajectory. We suggest that this pattern in the response dynamics is evidence for monitoring and provides further support for more controlled processing in nonfocal PM conditions (Abney et al., 2013). Furthermore, we found no quantitative differences between the focal condition and the control condition that would suggest monitoring in the focal condition. We did

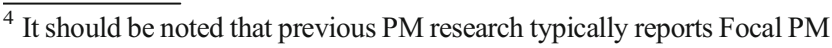
performance at $>90 \%$. As discussed in the Methods section, participants were allowed to ask questions about task instructions before moving on to the second block of the experiment. Furthermore, inspection of the experimental log for any comments from participants in the Focal PM condition did not indicate any instances of participants intending to press "z" but pressed "ENTER" instead. Therefore, the only explanation for the differences in Focal PM performance between the current study and reports from previous PM research is the modified design properties in the current study.
}

observe that the only velocity profile difference between the focal condition and the control condition was a higher velocity earlier in the trajectory (bins 8-9) for the focal condition. This observation was not expected but it is not evidence for monitoring because, as discussed above, monitoring would be evidenced by increased VEL later on in the response trajectory. One possibility is that the focal task provided facilitatory effects on ongoing task processing; however, a stronger claim for this possibility would require additional empirical support. The current results are not supportive of the PAM view (Smith, 2003) because this view proposes that successful PM performance requires attentional processing, such as monitoring, for all PM tasks. The results from the response dynamics provide more finegrained evidence supporting the notion that, for some PM tasks, monitoring may not be required for successful PM performance.

PM cue retrieval and cue interference

Our next conclusion concerns the dynamics of PM cue retrieval. In this analysis, we examined ongoing task responses to PM cues. Comparisons of IT and RT measures did not reveal any differences across PM cue retrieval trial type; however, there were nominal trends suggesting that the Focal $_{\text {HIT }}$ trials had faster ITs and slower RTs.

In the current study, we observed MAX $\mathrm{VEL}_{\mathrm{V}}$ occurring in later bins for Focal $_{\mathrm{HIT}}$ and Nonfocal $\mathrm{HIT}_{\mathrm{HT}}$ relative to

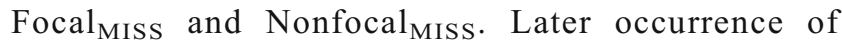
MAX $_{\mathrm{VEL}}$ in the response trajectory for $\mathrm{PM}_{\mathrm{HIT}}$ supports the prediction of cue interference because it suggests more interference of processing when a PM intention is active. Previous research observed cue interference through RT differences; however, in the current study we only observed differences in the response dynamics. One likely reason for this inconsistency is the difference in response actions (i.e., mouse-tracking vs. button press) between the present study and previous studies of cue interference of active PM intentions (Brewer et al., 2010; Marsh et al., 2002). 


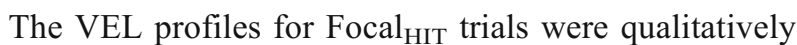
and quantitatively distinct relative to all other velocity profiles, including the Nonfocal $\mathrm{HIT}_{\mathrm{HT}}$ VEL profiles. There are a few conclusions we can draw from this result. First, the differences between Focal $_{\text {HIT }}$ and Nonfocal ${ }_{\text {HIT }}$ VEL profiles, along with the overall result that $\mathrm{MAX}_{\mathrm{VEL}}$ occurred in later bins for these PM trial types relative to $\mathrm{PM}_{\mathrm{MISS}}$ trials, suggest differences in cue interference as a function of cue focality. A focal PM intention might create more interference when noticing a target is a PM cue relative to a nonfocal PM intention. This possibility is supported by work suggesting that cuefocused discrepancy attribution processing of the PM cue slows processing when holding a PM intention (McDaniel et al., 2004). Given that the focal trials showed no evidence of monitoring, spontaneous retrieval through cue-focused discrepancy attribution is a likely process supporting successful PM retrieval in this condition. The attribution process may have resulted in a later $\mathrm{MAX}_{\mathrm{VEL}}$ in this condition due to the activation of these processes in spontaneously retrieving the PM intention. If so, the increased cue interference during the noticing of a focal PM cue would suggest that spontaneous retrieval is not wholly an automatic process (McDaniel \& Scullin, 2010). However, these conclusions are speculative given that this is the first examination (we are aware of) of the response dynamics for $\mathrm{PM}$ cue trials and requires further corroboration in future studies.

\section{Conclusions}

The current study provides novel insights into the debate regarding the processes that underlie successful PM performance by introducing the analysis of response dynamics into the PM literature. The observation of different response dynamics as a function of cue focality, even when controlling for the canonical measure of RT, supports the MP view of PM. Furthermore, the analysis of response dynamics provides a unique perspective into PM cue retrieval, and provides additional evidence for a cue interference effect as a function of cue focality. The introduction of response dynamics into the PM literature has the potential to expand current descriptions of PM performance with regard to mechanisms that support PM performance by adopting the emerging view that cognition and action are continuous.

\section{References}

Abney, D.H., McBride, D.M., \& Petrella, S.N. (2013). Interactive effects in transfer-appropriate processing for event-based prospective memory: The roles of effort, ongoing task, and PM cue properties. Memory \& Cognition, 41(7), 1032-1045.

Brewer, G. A., Knight, J. B., Marsh, R. L., \& Unsworth, N. (2010). Individual differences in event-based prospective memory: Evidence for multiple processes supporting cue detection. Memory \& Cognition, 38, 304-311.

Dale, R., Kehoe, C., \& Spivey, M. J. (2007). Graded motor responses in the time course of categorizing atypical exemplars. Memory \& Cognition, 35(1), 15-28.

Einstein, G. O., \& McDaniel, M. A. (2005). Prospective memory: Multiple retrieval processes. Current Directions in Psychological Science, 14, 286-290.

Einstein, G. O., \& McDaniel, M. A. (2010). Prospective memory and what costs do not reveal about retrieval processes: A commentary on Smith, Hunt, McVay, and McConnell (2007). Journal of Experimental Psychology: Learning, Memory, and Cognition, 36(4), 1082-8.

Freeman, J. B., \& Ambady, N. (2010). MouseTracker: Software for studying real-time mental processing using a computer mousetracking method. Behavior Research Methods, 42, 226-241.

Freeman, J., Dale, R., \& Farmer, T. (2011). Hand in motion reveals mind in motion. Frontiers in Psychology, 2, 59.

Kučera, H., \& Francis, W. N. (1967). Computational analysis of presentday American English. Providence, RI: Brown University Press.

Marsh, R. L., Hicks, J. L., \& Watson, V. (2002). The dynamics of intention retrieval and coordination of action in event-based prospective memory. Journal of Experimental Psychology: Learning, Memory, and Cognition, 28, 652-659.

McDaniel, M. A., \& Einstein, G. O. (2000). Strategic and automatic processes in prospective memory retrieval: A multiprocess framework. Applied cognitive psychology, 14(7), S127-S144.

McDaniel, M. A., \& Einstein, G. O. (2007). Prospective memory: An overview and synthesis of an emerging field. Thousand Oaks, CA: Sage.

McDaniel, M. A., \& Scullin, M. K. (2010). Implementation intention encoding does not automatize prospective memory responding. Memory \& Cognition, 38(2), 221-232.

McDaniel, M. A., Guynn, M. J., Einstein, G. O., \& Breneiser, J. (2004). Cue-focused and reflexive-associative processes in prospective memory retrieval. Journal of Experimental Psychology: Learning, Memory, and Cognition, 30(3), 605.

Papesh, M. H., \& Goldinger, S. D. (2012). Memory in motion: Movement dynamics reveal memory strength. Psychonomic Bulletin \& Review, 19(5), 906-913.

Rastle, K., Harrington, J., \& Coltheart, M. (2002). 358,534 nonwords: The ARC Nonword Database. Quarterly Journal of Experimental Psychology, 55A, 1339-1362.

Scullin, M. K., Einstein, G. O., \& McDaniel, M. A. (2009). Evidence for spontaneous retrieval of suspended but not finished prospective memories. Memory \& Cognition, 37, 425-433.

Scullin, M. K., McDaniel, M. A., \& Shelton, J. T. (2013). The Dynamic Multiprocess Framework: Evidence from prospective memory with contextual variability. Cognitive Psychology, 67(1), 55-71.

Smith, R. E. (2003). The cost of remembering to remember in eventbased prospective memory: Investigating the capacity demands of delayed intention performance. Journal of Experimental Psychology: Learning, Memory, and Cognition, 29, 347-361.

Smith, R. E. (2010). What costs do reveal and moving beyond the cost debate: Reply to Einstein and McDaniel (2010). Journal of Experimental Psychology: Learning, Memory, and Cognition, 36, 1089-1095. 